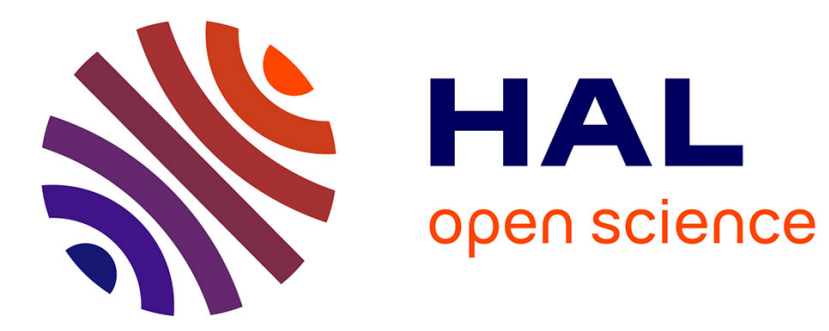

\title{
Pour un enseignement de la grammaire minimal et suffisant
}

Danièle Manesse

\section{To cite this version:}

Danièle Manesse. Pour un enseignement de la grammaire minimal et suffisant . Le Français Aujourd'hui, 2008, p.103-115. hal-01486619

\section{HAL Id: hal-01486619}

\section{https://hal-univ-paris3.archives-ouvertes.fr/hal-01486619}

Submitted on 10 Mar 2017

HAL is a multi-disciplinary open access archive for the deposit and dissemination of scientific research documents, whether they are published or not. The documents may come from teaching and research institutions in France or abroad, or from public or private research centers.
L'archive ouverte pluridisciplinaire HAL, est destinée au dépôt et à la diffusion de documents scientifiques de niveau recherche, publiés ou non, émanant des établissements d'enseignement et de recherche français ou étrangers, des laboratoires publics ou privés. 


\section{Danièle Manesse (2007), Le français aujourd'hui $n^{\circ} 162$, «Pour un enseignement de la grammaire minimal et suffisant "}

Quel flot, celui des textes officieux ou officiels sur la grammaire scolaire qui s'enchainent, qu'ils soient partie d'un programme complet ou non, chacun désavouant celui qui le précédait! Pour l'enseignement primaire : 1972, 1985, 1995, 2002, 2006 (rapport sur la grammaire dit de Bentolila), 2008; pour le collège 1977, 1985, 1996, 2004, sans doute 2008... Leur récurrence improductive montre que rien n'est jamais réglé. Elle atteste aussi les tensions très fortes qui s'exercent sur le français, et pour ce qui nous occupe, sur l'acquisition de la norme écrite.

Ceux qui ont une légitimité de fait à travailler sur la langue, les linguistes, exercent une pression certaine sur les programmes. Experts patentés, ils n'ont cessé de dénoncer les procédés et les règles que s'est bidouillés l'école pour enseigner la langue. Des linguistes de premier plan ont proposé des contenus alternatifs à ceux enseignés à l'école : dans les années 1970-80, les descriptions de la morphologie verbale de Martinet, à peine transposées, passaient de l'université dans les collections scolaires ; les manuels se couvraient d'arbres venus de la grammaire générative et transformationnelle, la grammaire sémantique de Charaudeau réactualisait les théories de Bally. Plus récemment, les programmes qui ont profondément transformé l'enseignement du français au collège depuis 1996 s'appuient ouvertement sur les "progrès des sciences du langage »: ils empruntent à Benveniste pour l'énonciation, à Combettes pour les progressions thématiques, la grammaire textuelle scolaire se nourrit de travaux d'Adam, etc.

Ces mouvements de transferts se sont faits dans une sorte d'évidence admise : l'idée qu'il serait plus aisé pour les élèves et les professeurs de se mouvoir dans un savoir grammatical (au sens large) construit à partir de descriptions de la langue et des usages de la langue plus cohérentes et plus conformes aux usages réels. En somme, nous avons maintenant intériorisé l'idée que des programmes et une démarche inspirés de la linguistique ou de la sociolinguistique permettent de meilleures acquisitions grammaticales. S'il parait difficile de ne pas partager cette conviction, on peut voir surgir des difficultés dans sa mise en œuvre.

C'est l'une d'elles que je voudrais discuter ici : à mon avis, la critique «scientifique » de la grammaires scolaire par des chercheurs, universitaires en général, a eu pour effet de construire une conception beaucoup trop exigeante de la grammaire scolaire; l'évolution de l'enseignement grammatical dans les programmes récents est marquée par la dissolution des objectifs les plus simples dans d'autres objectifs qui entraînent nécessairement des savoirs plus complexes, des démarches plus compliquées.

Dans un rapport sur le français au collège où depuis 1996 est mise en oeuvre la rénovation radicale des programmes autour de la maitrise des dicours, l'Inspection générale observait la difficulté à intégrer l'ancien et le nouveau: "A la question «que doit-on enseigner au collège » les professeurs répondent d'abord par une liste de notions parmi lesquelles beaucoup sont ressenties comme nouvelles, donc comme inquiétantes (grammaire de phrase, de texte, de discours $[\ldots .$.$] ; point de vue, implicite, narrateur, énonciation, modalisation [...]. La$ question des objectifs et des finalités leur semble beaucoup moins claire et beaucoup moins importante que celle des contenus et des méthodes ». L'Inspection générale rappelle alors les finalités «qui sont systématiquement développées en tête du programme de chaque classe », dont la première est « s'exprimer correctement et clairement, oralement et par écrit » ${ }^{1}$. C'est là une finalité qu'on peut trouver raisonnable, et je la prendrai pour admise.

\footnotetext{
${ }^{1}$ Inspection générale de l'éducation nationale, L'enseignement du français au collège, rapport à monsieur le ministre de l'éducation nationale et de la recherche, n²002-046, septembre 2002.
} 
Dans nombre de pays, la tradition scolaire n'accorde que peu d'importance dans les programmes scolaires à l'enseignement grammatical. Si en France, il a fallu beaucoup d'efforts pour simplifier la lourde machinerie grammaticale scolaire traditionnelle, cela n'impliquait pas qu'il faille compliquer les choses et lui substituer un modèle certes mieux fondé théoriquement, mais tout aussi pesant. Il me semble qu'on est parvenu à une situation paradoxale : d'une certaines manière "rénovateurs» et "traditionnalistes » partagent la même position, ils surestiment l'importance de l'enseignement de la grammaire. Je proposerai donc qu'on la limite à un strict minimum utile dans l'école obligatoire, avant d'en faire un objet de spéculation et de réflexion avec des élèves plus avancés en âge et déjà pourvus d'un bagage grammatical indispensable.

\section{La grammaire : un enseignement inefficace et donc injuste}

«Plus l'école sera efficace, plus elle sera juste. On doit cette efficacité aux élèves les plus défavorisés ${ }^{2}$ " martèle François Dubet. Si l'école est impuissante à transmettre un savoir, ceux des élèves qui n'ont pas de relais (parents, cours particuliers) pour l'acquérir ne l'acquerront pas. L'enseignement de la grammaire est de ceux-là : c'est un enseignement inefficace, donc injuste.

Le savoir-clé de l'école, la maitrise de la langue écrite, vecteur des apprentissages et condition des études longues, est incontestablement dépendant d'un certain nombre de connaissances grammaticales. Or celles-ci sont inégalement réparties : des mesures confirment obstinément le dénuement des élèves de classes populaires en matière de norme linguistique à l'écrit. Une recherche récente montre que la dégringolade de l'orthographe grammaticale est la cause du recul notable des performances des élèves de l'école obligatoire en matière d'orthographe, et qu'elle est bien plus accusée en moyenne chez les élèves de $\mathrm{ZEP}^{3}$. Ces résultats s'inscrivent dans un tableau connu : les élèves les plus défavorisés sont ceux qui ont la maitrise la moins grande des «outils de la langue», ponctuation, morphologie nominale et verbale (conjugaisons), orthographe grammaticale ; et c'est là que se creusent les plus grands écarts entre les élèves des ZEP et ceux du système ordinaire ${ }^{4}$. Rien d'étonnant : pour ceux qui ne la trouvent pas dans les pratiques familiales quotidiennes, pour ceux dont les usages oraux sont les plus éloignés des modèles de l'écrit, l'acquisition de la norme écrite est bien plus coûteuse. Si l'école ne prend pas la peine de "donner autant à ceux qui ont moins ", comme dit François Dubet, elle ne fait que perpétuer une inégalité. Il ne s'agit pas seulement de l'acquisition de l'emploi légitime de la langue: les connaissances grammaticales interviennent dans l'ensemble des activités écrites ; André Ouzoulias, citant Laurence Rieben («apprendre à orthographier, c'est apprendre à lire...»), rappelle qu'un enseignement inefficace de l'orthographe a des conséquences sur la lecture :

«[Certains] dont je suis, considèrent que cette baisse des performances en orthographe doit alerter les enseignants du primaire, car non seulement elle est mesurée par des études irréprochables, mais elle est très significative et elle commence à atteindre l'orthographe lexicale. Or, l'orthographe est déterminante... en lecture ! Si on ne fait pas la différence entre on et ont, entre sept et cette, entre toi et toit, etc. (orthographe lexicale), et si on ne distingue pas entre elle chante et elles chantent (orthographe grammaticale), c'est le sens qui est atteint. »

\footnotetext{
${ }^{2}$ François Dubet : « Donner autant à ceux qui ont moins »Cahiers pédagogiques n ${ }^{429-430}$ - Janvier-Février 2005

${ }^{3}$ Danièle Manesse, Danièle Cogis L'orthographes : à qui la faute, Paris ESF 2007 ;

Danièle Manesse, «Contre l'abandon orthographique des élèves de ZEP », Diversité, n¹51, SCEREN-CNDP, 2007.

${ }^{4}$ Voir sur ce point la discussion argumentée dans Danièle Manesse (dir.)Le français dans les classes difficiles, INRP Paris 2003, chapitre 2, p. 31-71
} 
Dans ce texte où il déplore que les décideurs s'aventurent à fabriquer de nouveaux programmes au lieu «de clarifier les recommandations faites en 2002 aux enseignants », il formule un programme minimum d'étude de la langue pour l'école, appuyé sur des pratiques systématiques : ce programme définirait « les compétences, attendues en fin de cycle 3, [qui] nécessitent des activités de systématisations, notamment dans l'analyse de phrase et l'orthographe grammaticale $\gg .^{5}$

\section{La sociolinguistique au secours de la grammaire ?}

Parmi les savoirs scolaires, la grammaire de l'école est un objet scolaire particulier, une construction à double fond. C'est un ensemble de règles et de procédures, donc de savoirs déclaratifs, dont André Chervel, par exemple, a expliqué la genèse dans différents ouvrages ${ }^{6}$; ces savoirs portent-ils sur un objet lui-même repérable et indiscutable : la langue ? Non, ce n'est pas « la » langue, mais le « bon usage », le « français standard », la « langue légitime », enfin cet ensemble construit. Ceci est maintenant une banalité, mais elle est de grande conséquence. Le didacticien de biologie qui met en question la manière d'enseigner le processus de la digestion ne met pas en question la réalité de la digestion; le didacticien du français comme on l'a si souvent dit et écrit, enseigne une fiction abstraite, la norme, objet introuvable, impossible à fixer par nature puisqu'il n'existe que dans la réalisation d'une infinité d'usages.

L'école peut-elle, à l'image du linguiste qui décrit, enseigner cet objet introuvable ? Une voie inspirée de la sociologie se présente, que suggère l'appel à contributions de ce numéro. On y formule l'espoir qu'une rénovation d'une didactique de la grammaire fondée sur l'observation des variantes pourrait permettre aux élèves « d'apprivoiser » la langue :

«Il serait possible de construire une didactique de la langue[qui s'appuierait ]sur des descriptions de la langue qui soient dans la proximité des locuteurs afin de faciliter l'apprentissage du standard, qui est l'objectif[...]D'une manière générale, c'est la descriptionmodélisation de la langue qu'il convient d'une part d'interroger et de renouveler en prenant en compte les travaux sur le non standard, à l'oral comme à l'écrit, et d'autre part d'en modifiant les approches avec les élèves. En effet, il faudrait vérifier si, en s'appuyant sur les usages des élèves, et par une approche contrastive, en comparant ces usages avec ceux du standard, on ne diminue pas la distance avec ce standard, le but étant d'amener les élèves à apprivoiser la langue ».

Le pouvoir de séduction d'une telle voie repose sur le fait, qui semble incontestable, qu'il convient d'enseigner des choses réelles, la réalité de la langue, celle de ses usages. Au lieu d'imposer une langue étrange et étrangère aux élèves, on va la leur faire construire, travailler avec le matériau qu'ils apportent la langue qu'ils emploient, leurs usages ; on voit se profiler l'image apaisée d'une pédagogie de la norme qui fera le détour par les pratiques des élèves, et leur permettra de se motiver et d'être les acteurs de leur acquisition. Je pense que cette proposition relève de la générosité, d'une position morale qui consiste à laisser la place à l'autre, au «divers », mais à mon sens, elle a peu de chances de permettre aux élèves qui en sont éloignés de s'approprier la norme utile.

Une telle proposition s'inscrit dans une tradition : l'attention à la variation n'est pas une nouveauté dans l'enseignement de la langue. La notion de registre de langue, celle de situation de communication, issues des sciences du langage sont apparues depuis les années 70, ont été transposées pour l'école, et sont à présent naturalisées dans les têtes enseignantes et les manuels scolaires. La démarche qui consisterait à prendre en compte dans la classe les formes non-standard est plus audacieuse : ce qui va être demandé aux élèves, c'est d'être les descripteurs d'un corpus à construire à chaque moment, à la suite de quoi ils pourront

\footnotetext{
${ }^{5}$ André Ouzoulias « M. Darcos, maître en déclinologie » le Café pédagogique, site internet, 28 mai -2008.

${ }^{6}$ Historie de la grammaire scolaire, Paris 1977 ; Histoire de l'enseignement du français du XIXème au XXème siècle, Retz, Paris 2007.
} 
s'approprier, moins brutalement et plus efficacement espère-t-on, les normes du français standard, dont l'enseignant est le garant. A partir de formes le plus souvent orales, ils travailleront comme des linguistes, en somme, allant en quelque sorte de la variation orale au système conservé dans l'écrit. Est-ce là une issue aux difficultés de l'enseignement de la grammaire?

\section{Travailler en classe la variation, l'acquisition de la norme sans douleur?}

Est-ce un chemin pertinent pour acquérir le français standard de prendre appui sur les productions langagières des élèves qui en sont le plus éloignés, et en procédant par approches contrastives ? C'est tout sauf certain.

Comment déjà surmonter cet écueil méthodologique qui guette le linguiste variationniste: le caractère non systématique et hasardeux du choix et du classement des variantes :

«[...] Ceux qu'inspirent les analyses sociolinguistiques visant à privilégier les données issues des productions «vernaculaires »[ ...] s'efforcent[...] de récupérer dans le corpus qui va leur servir de base de description, des faits qui étaient écartés par les grammairiens; ce qu'il y a de commun à toutes ces récupérations, c'est leur caractère non systématique ; les faits de langue non normatifs, notamment les données orales, sont pris en compte au coup par coup, sous formes d'exemples pris à la volée ou reconstitués après une discussion métalinguistique [. .. ] On peut bien ainsi constater que les linguistes ne prennent pas la peine de distinguer entre fait de langue non normatif et hapax non normatif....Une telle démarche aboutit à tenir pour établies des données comme : celui à qui que j'ai parlé l'homme que je l'ai vu, alors qu'il n'est pas sûr du tout sûr qu'elles soient attestées avec une quelconque régularité dans des usages non $\operatorname{standard~}^{7} \gg$.

Le linguiste dans la liberté de son étude n'est pas le professeur devant sa classe, dans les limites de son horaire. Que faire devant un amoncellement de variantes complaisamment proposées « au coup par coup » par des élèves d'autant plus productifs que la norme leur est étrangère ? Comment choisir, n'être pas débordé ? La situation risque d'être pédagogiquement ingérable, pour un professeur qui doit tenir un rôle de chercheur dans une situation qui ne le permet pas. Lui n'est pas nécessairement linguiste, et s'il l'est, il n'est pas dans la situation du linguiste lorsqu'il travaille avec des élèves jeunes : le linguiste a son temps, son droit au tâtonnement, son droit à l'aporie, au résultat provisoire. Le professeur est dans une obligation de résultats. L'étape de recherche de la forme normée avec les élèves n'a qu'un temps et ne peut se clore sur une interrogation... Suivant l'exemple que propose Sonia Branca dans un article récent, proposons dans une classe de ZEP un travail sur la morphologie du verbe ; soyons le maitre qui cherche à faire acquérir un point de morphologie verbale, le futur du verbe boire.

«Un linguiste qui prend le système comme un niveau explicatif, dit-elle, pourra ainsi rapporter les variations entre je boirai, je boiverai, je boivrai à une règle de formation du futur couvrant ces trois variantes : à partir du groupe phonétique $V+\mathrm{R}$, on peut produire le groupe plus régulier $\mathrm{v}+$ voyelle par insertion d'un $\mathrm{E}$ ou $\mathrm{R}$ voyelle par effacement $\mathrm{du} \mathrm{V}$. Puis, on constate que dans la communauté française seul boirai est la forme valorisée. Dans beaucoup de cas, évidemment, la situation est moins claire et dépend du modèle que se donne le grammairien $^{8} \gg$.

Mais que feront les élèves, sinon trouver les formes également plausibles, puisque les uns ou les autres les auront produites ? En dernière instance, eux vont devoir non constater mais

\footnotetext{
${ }^{7}$ José Deulofeu et André Valli « Sur l'aspect normatif des descriptions en français », in Gilles Siouffi et Agnés Steuckard, (éds)Les linguistes et la norme, aspects normatifs du discours linguistique, Peter Lang, Neuchâtel, 2007.
}

\footnotetext{
${ }^{8}$ Sonia branca Normes et genres de discours in Langage et société $n^{\circ} 119$, mars 2007, p. 112
} 
apprendre que « dans la communauté française seul boirai est la forme valorisée». A la vérité, qu'auront-ils gagné, sinon le risque de ne plus savoir, le moment venu, laquelle de ces formes est celle qu'ils doivent employer?

Pourquoi sans cesse reculer le moment où l'on dévoile la forme normée ? Pourquoi compliquer inutilement un chemin dont les élèves ont très tôt conscience : la langue écrite sélectionne dans les usages, et il y a une seule forme écrite de manière générale dans la langue qu'on emploie dans les livres et à l'école. Que le linguiste prenne le risque de passer à côté d'une description pertinente de l'usage, passe encore, il a mille moyens de s'en remettre. Que les enseignants risquent d'être en situation avec les élèves de se perdre dans la profusion des propositions et de n'avoir comme recours en dernière instance que d'imposer aux élèves " la forme » après avoir fait le détour d'une démarche ouverte et tolérante, n'est-ce pas les mettre dans une situation impossible?

Cela ne veut évidemment pas dire que les maitres n'aient pas à connaitre les usages des élèves : la conscience de la multiplicité des variantes et la capacité à les décrire est capitale, mais selon moi, elle est du côté du savoir du maitre ; elle lui permet anticipation et lucidité dans le travail d'enseignement de la norme, elle l'arme pour organiser son cours en fonction des élèves qu'il a devant lui. En cela, la grammaire partage le sort de toutes les disciplines, parce que les usages non normés des élèves, en quelque sorte, sont un réservoir de leurs conceptions préalables dont le professeur doit tenir compte pour savoir ce qui va faire obstacle à leur acquisition de la forme standard. Le futur de boire n'est ni *boivrai ni *buvrai, et il ne se discute pas, il s'apprend. Ce qui est important pour les élèves, c'est d'être au clair sur ce qu'est la norme. Et ce qui importe pour l'enseignant, c'est d'être au clair sur la procédure la plus économique pour enseigner la morphologie du futur.

Voici longtemps que Jean-Paul Bronckart critiquait l'illusion selon laquelle « la linguistique serait apte, en elle-même, à résoudre les problèmes pédagogiques liés au choix des programmes, comme à celui du modèle grammatical ou des exercices. Il s'agit là manifestement, d'une surestimation des possibilités de la linguistique et d'une sous-estimation de la complexité des enjeux pédagogiques ${ }^{9}$ ». On était alors au temps où la « linguistique appliquée » se combinait avec de nouvelles formes didactiques : l'enfant actif, motivé, dont l'expression personnelle est sollicitée.

« Aux niveaux de scolarité élémentaire, notamment, les méthodes ont perdu de leur rigidité et de leur mécanisme, et l'accent porté sur l'expression, l'action et la motivation de l'enfant a engendré des attitudes pédagogiques plus libres.[...] Mais, poursuivait-il, les résultats scolaires n'ont pas été à la mesure des espoirs placés dans la rénovation. Loin de disparaître, l'échec scolaire s'est apparemment accru, et les études entreprises soulignent le rôle sélectif que continue de jouer l'enseignement de la langue, en particulier celui de la grammaire. Comment expliquer ce paradoxe?

Il soulignait l'illusion qu'il y avait à croire qu'on peut rendre compatibles des objectifs normatifs, ceux de toujours dans l'école - et des objectifs de libération de la personne :

«Les rénovateurs semblent avoir cru qu'en se centrant sur « l'activité vraie » de l'enfant, ils pouvaient faire l'économie d'une réflexion sur le rôle des finalités traditionnelles; ils ont de cette manière sous-estimé la force et la permanence d'objectifs contradictoires avec la démarche qu'ils proposaient (norme et reproduction sociale, textes de la langue littéraire maintien du système orthographique) ....Dans ces conditions, les finalités nouvelles, relatives à l'enfant et à ses besoins, ne pouvaient que s'agglutiner aux précédentes Cette agglutination

\footnotetext{
${ }^{9}$ Jean-paul Bronckart Les sciences du langage, un défi pour l'enseignement, Delachaux et Niestlé UNESCO, 1985, Paris, chapitre 8 « Du bon usage des sciences du langage ».p. 105 sq, .
} 
inconsciente a pris, en réalité, l'allure d'une naturalisation de tous les objectifs ; les élèves d'aujourd'hui réfléchissent sur le langage (« ils font de la grammaire») pour garder le contact avec la pensée et la culture occidentale, pour sauver l'orthographe, mais aussi pour mieux communiquer oralement, pour mieux écrire et pour libérer leur personnalité ! C'est évidemment trop demander à l'enseignement d'une seule matière.»

La sociologue Bernadette Wynants met à jour elle aussi l'écartèlement et le difficile sentiment d'échec des professeurs de français, «partagés entre la volonté de favoriser une acquisition efficace du code et un souci d'intégrer cet apprentissage dans une démarche de pédagogie active $^{10} »$.Et on trouverait bien d'autres témoignages des difficultés à exercer le métier de professeur de français, placés dans cette double injonction.

Il est de l'essence de la norme linguistique d'être normative, et une, ainsi que d'être pour partie arbitraire - imprévisible ; elle doit être enseignée comme telle, sans dissimulation sur sa nature et sa fonction. Ce qu'apporte la linguistique, et ce n'est pas rien, c'est la possibilité de faire de cet enseignement une activité intéressante : d'organiser la présentation des faits de morphologie, de syntaxe et d'orthographe de la manière la plus lisible, la mieux ordonnée, la plus intelligente.

\section{Pour une grammaire minimum à l'école}

Il y a quelques années, nous avions proposé à près de 300 élèves ${ }^{11}$ de collèges de la Seine-Saint-Denis un questionnaire de représentations, composé de treize questions ouvertes, portant sur la lecture, sur la production d'écrit et sur les apprentissages scolaires. Comme il est d'usage, nous avions regroupé leurs réponses selon un petit nombre de modalités ${ }^{12}$. On n'avait alors su que faire de leurs réponses à la question «A ton avis, à quoi sert la grammaire ?» : trop d'entre eux n'avaient pas répondu, et les réponses étaient trop dispersées. Examinant ces questionnaires de nouveau six ans après, je suis finalement convaincue de la clarté du jugement de ceux qui se sont exprimés. Voici la répartition en modalités des réponses des élèves des 4 niveaux du collège.

A ton avis, à quoi sert la grammaire* ?

\begin{tabular}{|c|c|c|}
\hline réponses regroupées par modalité & $\% 6-5$ & $\%$ 4-3 \\
\hline 1. à comprendre le sens & 5 & 10.5 \\
\hline $\begin{array}{l}\text { 2. à apprendre des « mots » nouveaux } \\
\text { (dérivés, formation des mots) }\end{array}$ & 3 & 6 \\
\hline 3. à améliorer l'orthographe & 11 & 2 \\
\hline $\begin{array}{l}\text { 4. à améliorer la langue } \\
\text { 5. (à être meilleur en français) }\end{array}$ & 7.5 & 10 \\
\hline 6. à écrire mieux & 18 & 11 \\
\hline 7. à lire mieux & 12 & 3.5 \\
\hline 8. à parler mieux & 4 & 3 \\
\hline 9. à connaître les temps des verbes & 5 & 8 \\
\hline 10. à connaître « $\mathrm{COD}$, fonctions $»$ etc & 5 & 11 \\
\hline
\end{tabular}

\footnotetext{
${ }^{10}$ « Orthographe française, altérations et crispations », in J. De Munck. et J. Verhoeven Les mutations du rapport à la norme. Un changement dans la modernité. De Boeck Université, 1997

${ }^{11}$ Danièle Manesse (dir.) Le français dans les classes difficiles,op.cit, 2003, chapitre 2.

${ }^{12}$ On trouvera en exemple les réponses à cette question des élèves d'une classe de troisième de Clichy-sous-bois.
} 


$\begin{array}{lll}\text { 11. à connaître la ponctuation } & 3 & 1 \\ \begin{array}{l}\text { 12. la grammaire sert à faire } \\ \text { des exercices de grammaire }\end{array} & 2 & 1 \\ \begin{array}{l}\text { 13. à rien, à compliquer } \\ \text { 14. autres }\end{array} & 1 & 6 \\ \text { 15. NR } & 16 & 21 \\ \end{array}$

*Les pourcentages sont arrondis au demi-point ; la première colonne donne les résultats pour les élèves de sixième et de cinquième, la seconde pour les élèves de quatrième et de troisième. Les élèves pouvaient donner plus d'une réponse ; les totaux sont donc supérieurs à 100\%.

Certes, il y $40 \%$ de réponses inclassables, tant elles sont divergentes, ou de non réponses. On pourrait trouver décourageant le peu de réponses évoquant la contribution de la grammaire à la compréhension du sens. En revanche, plus de 50\% des réponses disent bien ce qu'est la grammaire : un outil pour acquérir la langue écrite, pour lire mieux, pour maitriser la norme. Les élèves des petites classes du collège, plus proches de l'école primaire en sont davantage convaincus : au fil du collège décroît la confiance dans la grammaire pour améliorer la langue, pour lire, écrire, parler, et même les élèves les plus avancés ne sont que faiblement plus nombreux à voir que la grammaire contribue au sens. Ceci nous informe à mon avis sur le sens dans lequel il faudrait travailler.

\section{Pour conclure : pour une définition modeste de la grammaire à l'école obligatoire}

Quels sont en définitive les objectifs de la grammaire ? Assurons déjà le minimum utile, ce dont les élèves auront besoin dans leur scolarité. Trois points, pas plus, à mon avis :

(1) Que requiert l'orthographe morphosyntaxique ? Avant tout la connaissance des classes de mots, et celle de quelques fonctions (sujet, objet), des règles d'accord, des formes verbales.

(1) Que requièrent les activités de production écrite ? La capacité à enchainer et organiser les phrases, donc à connaître les temps, les connecteurs.

(1) Que requiert l'entrée dans les apprentissages des langues étrangères ? Un métalangage minimal, celui en premier lieu qui concerne les classes de mots et les noms des temps.

En l'état actuel des choses, bien des élèves n'ont pas ces maitrises au collège, comme en témoignent les études précitées, les bilans de l'inspection, des notes de la DEPP ...

Tant qu'elles ne sont pas installées, tant que les élèves trainent comme un boulet le problème de la norme qu'ils ne maitrisent pas, je pense que toutes les connaissances qui exigent des démarches de secondarisation, de mise à distance critique de la langue risquent d'être improductives : c'est le cas de la difficile théorisation des genres de discours, c'est le cas du travail sur la variabilité des formes qu'autorise le système du français. On ne peut les développer qu'avec des élèves qui jouissent déjà d'une sécurité linguistique minimale. Telle est en tout cas mon expérience dans les classes « difficiles ».

Danièle Manesse

Université Paris 3-Sorbonne nouvelle, DIFLE

Equipe de recherche DILTEC

A quoi sert la grammaire à ton avis? 
Classe de Troisième d'un collège de Clichy-sous-Bois, 22 élèves (2002)

(Orthographe conservée. J'ai ajouté un point après chaque proposition. NR : non réponse )

1. A savoir l'accord des verbes, comment est formé un mot.

2. A comprendre des mots et à le savoir.

3. A mieux construir nos phrases et parfois aussi à mieux nous exprimer.

4. NR

5. la grammaire sert à donner un sens à la phrase. Sens elle, les verbes seront mal conjuger, les phrases à l'écrit n'auront aucun sens.

6. Sa sert à bien conjugué les verbes, à connaître les fonctions et les natures de mots. A connaître les mots comment ils sont construits.

7. NR

8. la grammaire sert a mieux connaître les règles et a pouvoir les appliquer en orthographe.

9. La grammaire sert à apprendre les définitions de la langue Française.

10. La grammaire sert à mettre en évidence les différentes versions qu'un mot peut renconter.

11. la grammaire pour moi elle sert a donnée un sens au phrases.

12. je sais pas.

13. - A avoir un vocabulaire élevé

- dans la prononsiation orale

- et à ne pas faire de faute de grammaire.

14. La grammaire nous sert à nous faire connaître les determinant les adj. ect....pour écrire mieux.

15. la grammaire ça sert à compre un texte.

16. NR

17. Ca sert à savoir le sens, la nature, la fonct ${ }^{\circ}$ des mots d'une phrase.

18. A pouvoir faire des phrases correctes.

19. A savoir conjuguer.

20. NR

21. à apprendre a utiliser les mots approprié dans certaine condition.

22. a Mieux comprendre les Mots. 